\title{
A novel efficient homotopy continuation method in tracking
}

\author{
Yueh-Cheng Kuo, Wen-Wei Lin, And Shing-Tung Yau
}

Tracking a moving object by Doppler effect is an important tool to locate the position and to estimate the velocity of a moving object. Suppose that we have $N(N \geqslant 6)$ observation stations which measure the relative speed of the moving object by Doppler effect. Theoretically, the corresponding movement of the moving object can be formulated by a system of $2 N$ polynomials in $N+6$ unknowns. In this paper, we propose a novel simplification to reduce the original system to a system of $N-2$ polynomials in 4 unknowns. Then using this simplified system we develop an efficient homotopy continuation method to trace the moving object. Numerical experiments show that the position and the velocity of the moving object can be solved efficiently and reliably by the homotopy continuation method.

\section{Introduction}

Tracking a moving object by Doppler effect is an important subject in many applications $[2,3,10]$. Suppose that $\mathbf{u}(t)=(x(t), y(t), z(t))^{T}$ is the position of a moving object with respect to time $t$ and $\left\{\mathbf{s}_{j} \equiv\left(x_{j}, y_{j}, z_{j}\right)\right\}_{j=1}^{N}$ are locations of $N$ fixed observation stations. In general, the relative speeds of the moving object can be measured by Doppler effect by those $N$ observation stations. The distances, $r_{j}(t)$, between $\mathbf{u}(t)$ and $\mathbf{s}_{j}$ can be formulated as

$$
\left(x(t)-x_{j}\right)^{2}+\left(y(t)-y_{j}\right)^{2}+\left(z(t)-z_{j}\right)^{2}=r_{j}(t)^{2},
$$

$j=1, \ldots, N$. Differentiating (1) with respect to $t$, we obtain

$$
\left(x(t)-x_{j}\right) \dot{x}(t)+\left(y(t)-y_{j}\right) \dot{y}(t)+\left(z(t)-z_{j}\right) \dot{z}(t)=r_{j}(t) \dot{r}_{j}(t),
$$

$j=1, \ldots, N$. In practice, the datas $\left\{\dot{r}_{j}(t)\right\}_{j=1}^{N}$ in (2) are measured by Doppler effect. 
Suppose that the relative speeds of the moving object, $\left\{\dot{r}_{j}(t)\right\}_{j=1}^{N}$, are given in each time-step by those $N$ observation stations. From (1) and (2), it is easily seen the position of the object, $(x, y, z)$, and the associated velocity, $(\dot{x}, \dot{y}, \dot{z})$, satisfy a system of nonlinear equations $\mathbf{F}(\mathbf{v})=\mathbf{0}$, where $\mathbf{F} \equiv\left(F_{1}, \ldots, F_{N}\right): \mathbb{R}^{6} \rightarrow \mathbb{R}^{N}, \mathbf{v}=(x, y, z, \dot{x}, \dot{y}, \dot{z})^{T} \in \mathbb{R}^{6}$ with

$$
F_{j}(\mathbf{v})=\frac{\left(x-x_{j}\right) \dot{x}+\left(y-y_{j}\right) \dot{y}+\left(z-z_{j}\right) \dot{z}}{\sqrt{\left(x-x_{j}\right)^{2}+\left(y-y_{j}\right)^{2}+\left(z-z_{j}\right)^{2}}}-\dot{r}_{j} .
$$

Note that $\mathbf{F}(\mathbf{v})=\mathbf{0}$ has 6 unknowns. Naturally, we consider six $(N=6)$ measured datas $\left\{\dot{r}_{j}\right\}_{j=1}^{6}$, and hence, generically, the system of equations $\mathbf{F}(\mathbf{v})=\mathbf{0}$ has only isolated solutions. When $N<6$, the solution set of the system $\mathbf{F}(\mathbf{v})=\mathbf{0}$ is not isolated, hence, it is impossible to trace the moving object. In this paper, we consider the Doppler tracking with $N \geqslant 6$ observation stations.

To track the moving object, a homotopy continuation method has been proposed in [7] for computing all isolated solutions of polynomial equations of (1) and (2) with $N=6$ at each time $t$. For given measured datas $\left\{\dot{r}_{j}\right\}_{j=1}^{6}$, (1) and (2) have 12 equations and 12 unknowns $\left\{x, y, z, \dot{x}, \dot{y}, \dot{z}, r_{1}, \ldots, r_{6}\right\}$. In [7], authors first reduce those 12 polynomial equations to 4 polynomial equations in 4 unknowns $\left\{r_{3}, r_{4}, r_{5}, r_{6}\right\}$, and then, employ the homotopy method [1, 8] to compute all isolated solutions. If $\mathbf{x}=\left(r_{3}, r_{4}, r_{5}, r_{6}\right)^{T}$ is a solution of this reduced system, where $r_{3}, r_{4}, r_{5}$ and $r_{6}$ represent the distances between the moving object and the observation stations $\mathbf{s}_{3}, \mathbf{s}_{4}, \mathbf{s}_{5}$ and $\mathbf{s}_{6}$, respectively, then the position of the moving object, $(x, y, z)$, and the associated velocity, $(\dot{x}, \dot{y}, \dot{z})$, can be obtained by the solution $\mathbf{x}$. In general, the system of polynomial equations (1) and (2) with $N=6$ has 48 complex solutions and we are only interested in the real solution.

In this paper, we propose an efficient homotopy continuation method to trace the moving object with the observation stations $N \geqslant 6$. In Section 2 we reduce the system of $2 N$ equations (1) and (2) to a new system of $N-2$ equations in 4 unknowns. In Section 3 we develop two algorithms, one for the case $N=6$ and the other for the case $N>6$, to trace the moving object. The numerical experiments will be presented in Section 4. Finally, a conclusion is given in Section 5 .

Throughout this paper, we use bold face letters or symbols to denote matrices or vectors. $\mathbf{I}_{n}$ is defined by an identity matrix of order $n$ and $\mathbf{e}=$ $(1,1, \ldots, 1)^{T}$. For $\mathbf{v}=\left(v_{1}, v_{2}, \ldots, v_{n}\right)^{T}, \mathbf{w}=\left(w_{1}, w_{2}, \ldots, w_{n}\right)^{T}, \mathbf{v} \circ \mathbf{w}=$ $\left(v_{1} w_{1}, v_{2} w_{2}, \ldots, v_{n} w_{n}\right)^{T}$ denotes the Hadamard product of $\mathbf{v}$ and $\mathbf{w}$ and $[|\mathbf{v}|]:=\operatorname{diag}(\mathbf{v})$ denotes the diagonal matrix of $\mathbf{v}$. 


\section{Simplification of systems of polynomials}

In this paper, we consider the Doppler tracking with six or more than six observation stations $(N \geqslant 6)$. The original system of polynomial equations (1) and (2) has $2 N$ equations and $N+6$ unknowns $\left\{x, y, z, \dot{x}, \dot{y}, \dot{z}, r_{1}, \ldots, r_{N}\right\}$. Note that when $N>6$ the original system is an overdetermined system. In this section, we will propose a novel simplification to reduce the original system to a new system of $N-2$ equations in 4 unknowns.

For given $N$ positions $\left\{\left(x_{j}, y_{j}, z_{j}\right)\right\}_{j=1}^{N}$ of observation stations and the measured speeds $\left\{\dot{r}_{j}\right\}_{j=1}^{N}$ by Doppler effect, we denote

$$
\mathbf{V}_{0}=\left[\begin{array}{ccc}
x_{1} & y_{1} & z_{1} \\
x_{2} & y_{2} & z_{2} \\
\vdots & \vdots & \vdots \\
x_{N} & y_{N} & z_{N}
\end{array}\right], \dot{\mathbf{r}}=\left[\begin{array}{c}
\dot{r}_{1} \\
\dot{r}_{2} \\
\vdots \\
\dot{r}_{N}
\end{array}\right], \quad \mathbf{s}_{j}=\left[\begin{array}{c}
x_{j} \\
y_{j} \\
z_{j}
\end{array}\right]
$$

for $j=1,2, \ldots, N$. For the unknown values, we denote

$$
\mathbf{u}=\left[\begin{array}{c}
x \\
y \\
z
\end{array}\right], \dot{\mathbf{u}}=\left[\begin{array}{c}
\dot{x} \\
\dot{y} \\
\dot{z}
\end{array}\right], \quad \mathbf{r}=\left[\begin{array}{c}
r_{1} \\
r_{2} \\
\vdots \\
r_{N}
\end{array}\right] .
$$

Subtracting the 1 st equation from the $j$ th equation $(j \neq 1)$ in $(1)$ and (2), respectively, yield

$$
\begin{aligned}
& \mathbf{C V}_{0} \mathbf{u}=\frac{1}{2} \mathbf{C}(\mathbf{n}-\mathbf{r} \circ \mathbf{r}), \\
& \mathbf{C V}_{0} \dot{\mathbf{u}}=-\mathbf{C}[|\dot{\mathbf{r}}|] \mathbf{r}
\end{aligned}
$$

where

$$
\mathbf{n}=\left(\left\|\mathbf{s}_{1}\right\|^{2},\left\|\mathbf{s}_{2}\right\|^{2}, \ldots,\left\|\mathbf{s}_{N}\right\|^{2}\right)^{T}, \quad \mathbf{C}=\left[-\mathbf{e}, \mathbf{I}_{N-1}\right] \in \mathbb{R}^{(N-1) \times N}
$$

with $\mathbf{e}=(1, \ldots, 1)^{T}$. Suppose that $N$ observation stations are not on a plane. Then, it is easily seen that $\mathbf{C V}_{0}$ is of full rank and the matrix $\mathbf{V}_{0}^{T} \mathbf{C}^{T} \mathbf{C} \mathbf{V}_{0}$ is invertible. Note that if $\mathbf{C V}_{0}$ is of full rank, then so is $\mathbf{V}_{0}$. From (5) and (6) $\mathbf{u}$ and $\dot{\mathbf{u}}$ can be written as

$$
\mathbf{u}=\mathbf{A q}, \quad \dot{\mathbf{u}}=\mathbf{A p},
$$


where

$$
\begin{aligned}
& \mathbf{A}=\left(\mathbf{V}_{0}^{T} \mathbf{C}^{T} \mathbf{C V}_{0}\right)^{-1}\left(\mathbf{V}_{0}^{T} \mathbf{C}^{T} \mathbf{C}\right) \\
& \mathbf{p}=-\dot{\mathbf{r}} \circ \mathbf{r} \equiv\left(p_{1}, \ldots, p_{N}\right)^{T}, \quad \mathbf{q}=\frac{1}{2}(\mathbf{n}-\mathbf{r} \circ \mathbf{r})
\end{aligned}
$$

Equation (7) shows that the unknowns $\mathbf{u}$ and $\dot{\mathbf{u}}$ in (4) can be represented in terms of $\mathbf{q}$ and $\mathbf{p}$, respectively, and the vectors $\mathbf{q}$ and $\mathbf{p}$ are dependent on the unknowns $\left\{r_{j}\right\}_{j=1}^{N}$. Substituting (7) into (1) and (2), we obtain

$$
\mathbf{q}^{T} \mathbf{A}^{T} \mathbf{A q}-2 \mathbf{s}_{j}^{T} \mathbf{A q}+\mathbf{s}_{j}^{T} \mathbf{s}_{j}=\dot{r}_{j}^{-2} p_{j}^{2},
$$

and

$$
\mathbf{q}^{T} \mathbf{A}^{T} \mathbf{A} \mathbf{p}-\mathbf{s}_{j}^{T} \mathbf{A} \mathbf{p}=-p_{j}
$$

respectively, for $j=1, \ldots, N$.

Next, we show that those $N$ unknowns $\left\{r_{j}\right\}_{j=1}^{N}$ satisfy some relations. Subtracting the 2 nd equation from the $j$ th equation $(j=3, \ldots, N)$ in $(10)$, yields

$$
\left(\left[\begin{array}{c}
\left(\mathbf{s}_{2}-\mathbf{s}_{3}\right)^{T} \\
\left(\mathbf{s}_{2}-\mathbf{s}_{4}\right)^{T} \\
\vdots \\
\left(\mathbf{s}_{2}-\mathbf{s}_{N}\right)^{T}
\end{array}\right] \mathbf{A}+\widehat{\mathbf{C}}\right)(\dot{\mathbf{r}} \circ \mathbf{r}) \equiv \widehat{\mathbf{A}}(\dot{\mathbf{r}} \circ \mathbf{r})=\mathbf{0}
$$

where $\widehat{\mathbf{C}}=\left[\mathbf{0},-\mathbf{e}, \mathbf{I}_{N-2}\right]$ and $\widehat{\mathbf{A}} \in \mathbb{R}^{(N-2) \times N}$. In fact, the rank of $\widehat{\mathbf{A}}$ can be shown, generically, to be $N-4$ (see Appendix). In fact, if $\mathbf{s}_{2}+\mathbf{s}_{3}+\cdots+\mathbf{s}_{N} \neq$ $(N-1) \mathbf{s}_{1}$, then $\operatorname{rank}(\widehat{\mathbf{A}})=N-4$. Let $\widehat{\mathbf{A}}=\mathbf{Q}\left[\begin{array}{cc}\mathbf{T}_{1} & \mathbf{T}_{2} \\ \mathbf{0} & \mathbf{0}\end{array}\right]$ be the QR factorization of $\widehat{\mathbf{A}}$, where $\mathbf{T}_{1} \in \mathbb{R}^{(N-4) \times(N-4)}$ is invertible and $\mathbf{T}_{2} \in \mathbb{R}^{(N-4) \times 4}$. Suppose that $\dot{r}_{j} \neq 0$ for $j=1, \ldots, N-4$. It follows from (11) that

$$
\left[\begin{array}{c}
r_{1} \\
r_{2} \\
\vdots \\
r_{N-4}
\end{array}\right]=-\dot{\mathbf{R}}_{1}^{-1} \mathbf{T}_{1}^{-1} \mathbf{T}_{2} \dot{\mathbf{R}}_{2}\left[\begin{array}{c}
r_{N-3} \\
r_{N-2} \\
r_{N-1} \\
r_{N}
\end{array}\right]
$$

where $\dot{\mathbf{R}}_{1}=\operatorname{diag}\left(\dot{r}_{1}, \ldots, \dot{r}_{N-4}\right)$ and $\dot{\mathbf{R}}_{2}=\operatorname{diag}\left(\dot{r}_{N-3}, \ldots, \dot{r}_{N}\right)$. Hence, the unknown variables $r_{1}, \ldots, r_{N-4}$ can be represented in terms of variables $r_{N-3}, r_{N-2}, r_{N-1}$ and $r_{N}$. 
Substituting $\mathbf{p}$ and $\mathbf{q}$ in (8) into the equations (9) and (10) with $j=1$, yield

$$
\begin{aligned}
& \frac{1}{4}(\mathbf{r} \circ \mathbf{r})^{T} \mathbf{A}^{T} \mathbf{A}(\mathbf{r} \circ \mathbf{r})-\hat{\mathbf{s}}_{1}^{T} \mathbf{A}(\mathbf{r} \circ \mathbf{r})+\hat{c}=r_{1}^{2}, \\
& \frac{1}{2}(\mathbf{r} \circ \mathbf{r})^{T} \mathbf{A}^{T} \mathbf{A}(\dot{\mathbf{r}} \circ \mathbf{r})-\hat{\mathbf{s}}_{1}^{T} \mathbf{A}(\dot{\mathbf{r}} \circ \mathbf{r})=r_{1} \dot{r}_{1},
\end{aligned}
$$

where

$$
\hat{\mathbf{s}}_{1}=\frac{1}{2} \mathbf{A} \mathbf{n}-\mathbf{s}_{1}, \quad \hat{c}=\frac{1}{4} \mathbf{n}^{T} \mathbf{A}^{T} \mathbf{A} \mathbf{n}-\mathbf{s}_{1}^{T} \mathbf{A} \mathbf{n}+\mathbf{s}_{1}^{T} \mathbf{s}_{1} .
$$

Subtracting the 2 nd equation from the $j$ th equation $(j=3, \ldots, N)$ in (9), we obtain

$$
\left(\left[\begin{array}{c}
\left(\mathbf{s}_{2}-\mathbf{s}_{3}\right)^{T} \\
\left(\mathbf{s}_{2}-\mathbf{s}_{4}\right)^{T} \\
\vdots \\
\left(\mathbf{s}_{2}-\mathbf{s}_{N}\right)^{T}
\end{array}\right] \mathbf{A}+\widehat{\mathbf{C}}\right)(\mathbf{r} \circ \mathbf{r}-\mathbf{n}) \equiv \widehat{\mathbf{A}}(\mathbf{r} \circ \mathbf{r}-\mathbf{n})=\mathbf{0} .
$$

From the relation in (12), the equations (13) and (15) can be simplified to equations in variables $r_{N-3}, r_{N-2}, r_{N-1}$ and $r_{N}$. Since the matrix $\widehat{\mathbf{A}}$ is generically of rank $N-4$, we can choose $N-4$ linearly independent equations in (15), say the first $N-4$ equations.

Let $\left\{\mathbf{s}_{j}=\left(x_{j}, y_{j}, z_{j}\right)^{T}\right\}_{j=1}^{N}$ be $N$ positions of observation stations and $\left\{\dot{r}_{j}\right\}_{j=1}^{N}$ be the measured Doppler data. Then the distances between the moving object and the $N$ stations, $\left\{r_{j}\right\}_{j=1}^{N}$, satisfy the relation in (12), i.e., if $\mathbf{x} \equiv\left(r_{N-3}, r_{N-2}, r_{N-1}, r_{N}\right)^{T}$ is given, then $r_{1}, \ldots, r_{N-4}$ can be obtained by (12). The vector $\mathbf{x}$ should satisfy $N-2$ equations: $\widehat{\mathbf{G}}(\mathbf{x})=\mathbf{0}$, where $\widehat{\mathbf{G}}: \mathbb{R}^{4} \rightarrow \mathbb{R}^{N-2}$ and

$$
\widehat{\mathbf{G}}(\mathbf{x})=\left[\begin{array}{c}
\frac{1}{4}(\mathbf{r} \circ \mathbf{r})^{T} \mathbf{A}^{T} \mathbf{A}(\mathbf{r} \circ \mathbf{r})-\hat{\mathbf{s}}_{1}^{T} \mathbf{A}(\mathbf{r} \circ \mathbf{r})-r_{1}^{2}+\hat{c} \\
\frac{1}{2}(\mathbf{r} \circ \mathbf{r})^{T} \mathbf{A}^{T} \mathbf{A}(\dot{\mathbf{r}} \circ \mathbf{r})-\hat{\mathbf{s}}_{1}^{T} \mathbf{A}(\dot{\mathbf{r}} \circ \mathbf{r})-r_{1} \dot{r}_{1} \\
\left(\mathbf{s}_{2}-\mathbf{s}_{3}\right)^{T} \mathbf{A}(\mathbf{r} \circ \mathbf{r})-r_{2}^{2}+r_{3}^{2}+\hat{c}_{3} \\
\vdots \\
\left(\mathbf{s}_{2}-\mathbf{s}_{N-2}\right)^{T} \mathbf{A}(\mathbf{r} \circ \mathbf{r})-r_{2}^{2}+r_{N-2}^{2}+\hat{c}_{N-2}
\end{array}\right],
$$

where $\hat{\mathbf{s}}_{1}, \hat{c}$ are given in (14) and $\hat{c}_{j}=\mathbf{s}_{2}^{T} \mathbf{s}_{2}-\mathbf{s}_{j}^{T} \mathbf{s}_{j}-\left(\mathbf{s}_{2}-\mathbf{s}_{j}\right)^{T} \mathbf{A n}$ for $j=3, \ldots, N-2$.

Remark 2.1. Given $N$ positions of observation stations $\left\{\mathbf{s}_{j}\right\}_{j=1}^{N}$ and the measured Doppler data $\left\{\dot{r}_{j}\right\}_{j=1}^{N}\left(\dot{r}_{j} \neq 0\right.$, for $\left.j=1, \ldots, N\right)$ such that the 
first $N-4$ rows of the matrix $\widehat{\mathbf{A}}$ defined in (11) are linearly independent. It is easily seen that the system of equations (1) and (2) has solution $\left(x, y, z, \dot{x}, \dot{y}, \dot{z}, r_{1}, \ldots, r_{N}\right)$ if and only if $\left(r_{N-3}, r_{N-2}, r_{N-1}, r_{N}\right)$ is a solution of $\widehat{\mathbf{G}}(\mathbf{x})=\mathbf{0}$. Furthermore, $(x, y, z, \dot{x}, \dot{y}, \dot{z})$ is real if and only if $\left(r_{N-3}, r_{N-2}, r_{N-1}, r_{N}\right)$ is real.

Remark 2.2. In the case $N=6$, the system $\widehat{\mathbf{G}}(\mathbf{x})=\mathbf{0}$ can theoretically be simplified to a system of 2 polynomial equations of degree 16 and of degree 12 in 2 unknowns. The simplification method has been well developed in [7]. Compared to $\widehat{\mathbf{G}}(\mathbf{x})=\mathbf{0}$, the number of unknowns in the simplification system is reduced from 4 to 2 . However, the maximum of degrees amount all equations in the system is increased from 4 to 16 . In the case $N>6$, the overdetermined system $\widehat{\mathbf{G}}(\mathbf{x})=\mathbf{0}$ can be simplified to a system of $N-4$ polynomial equations in 2 unknowns by using the same simplification method, two of which are polynomials of degree 16 and of degree 12 , respectively. However, in the practical situation in numerical continuation, the 2-variable polynomial system with higher degrees is much more troublesome than $\widehat{\mathbf{G}}(\mathbf{x})=\mathbf{0}$.

When $N>6, \widehat{\mathbf{G}}(\mathbf{x})=\mathbf{0}$ is an overdetermined system. In general, $\widehat{\mathbf{G}}(\mathbf{x})=$ $\mathbf{0}$ has no solution due to Doppler effect. We can choose 4 equations of $\widehat{\mathbf{G}}(\mathbf{x})=$ $\mathbf{0}$, say $\mathbf{G}(\mathbf{x})=\mathbf{0}$, to compute its solutions, where $\mathbf{G}: \mathbb{R}^{4} \rightarrow \mathbb{R}^{4}$. The system of equations $\mathbf{G}(\mathbf{x})=\mathbf{0}$ have many solutions, e.g., if $\mathbf{G}(\mathbf{x})=\mathbf{0}$ is the system of the first 4 equations of $\widehat{\mathbf{G}}(\mathbf{x})=\mathbf{0}$, then, generally, it has 48 solutions (see $[7,8])$. Because $\left\{\dot{r}_{j}\right\}_{j=1}^{N}$ are the measured speeds of a moving object, generically, there is only one real solution $\mathbf{x}_{*}$ of $\mathbf{G}(\mathbf{x})=\mathbf{0}$ such that the residual $\left\|\widehat{\mathbf{G}}\left(\mathbf{x}_{*}\right)\right\|$ has the same order with the error of measured data. Next, we define a desired solution of $\mathbf{G}(\mathbf{x})=\mathbf{0}$.

Definition 2.3. Let $\mathbf{G}(\mathbf{x})=\mathbf{0}$ be a system of 4 equations chosen from $\widehat{\mathbf{G}}(\mathbf{x})=\mathbf{0}$. Then $\mathbf{x} \in \mathbb{R}^{4}$ is called a desired solution of $\mathbf{G}(\mathbf{x})=\mathbf{0}$ if $\mathbf{x}$ is a regular solution of $\mathbf{G}(\mathbf{x})=\mathbf{0}$ such that the residual $\|\widehat{\mathbf{G}}(\mathbf{x})\|$ attains its minimum.

If $\mathbf{x}$ is the desired solution of $\mathbf{G}(\mathbf{x})=\mathbf{0}$, then the position $\mathbf{u}=(x, y, z)^{T}$ and the velocity $\dot{\mathbf{u}}=(\dot{x}, \dot{y}, \dot{z})^{T}$ of the moving object can be obtained by using formulas (12) and (7).

\section{Homotopy continuation methods}

In this section, we develop an efficient algorithm to trace the moving object. Let $\left\{\mathbf{s}_{j}\right\}_{j=1}^{N}$ be $N(N \geqslant 6)$ positions of observation stations such that 
$\mathbf{s}_{2}+\mathbf{s}_{3}+\cdots+\mathbf{s}_{N} \neq(N-1) \mathbf{s}_{1}$. Then $\operatorname{rank}(\widehat{\mathbf{A}})=N-4$ (see Appendix), where $\widehat{\mathbf{A}}$ is defined in (11). Let the first $N-4$ rows of the matrix $\widehat{\mathbf{A}}$ be linearly independent. Suppose that $\left\{\dot{r}_{j}^{0} \equiv \dot{r}_{j}\left(t_{0}\right)\right\}_{j=1}^{N}$ and $\left\{\dot{r}_{j}^{1} \equiv \dot{r}_{j}\left(t_{1}\right)\right\}_{j=1}^{N}$ are measured speeds of a moving object by Doppler effect from those $N$ stations at time $t_{0}$ and $t_{1}$, respectively, where $t_{0}<t_{1}$ and $t_{0} \simeq t_{1}$. Let $\widehat{\mathbf{G}}^{0}(\mathbf{x})$ and $\widehat{\mathbf{G}}^{1}(\mathbf{x})$ be given in (16) with parameters $\dot{r}_{j}=\dot{r}_{j}^{0}$ and $\dot{r}_{j}=\dot{r}_{j}^{1}$, respectively. Suppose that $\mathbf{x}_{0} \equiv\left(r_{N-3}^{0}, r_{N-2}^{0}, r_{N-1}^{0}, r_{N}^{0}\right)^{T}$ is a desired solution of $\mathbf{G}^{0}(\mathbf{x})=\mathbf{0}$, where $\mathbf{G}^{0}(\mathbf{x})=\widehat{\mathbf{E}}^{T} \widehat{\mathbf{G}}^{0}(\mathbf{x})$ and $\widehat{\mathbf{E}} \in \mathbb{R}^{(N-2) \times 4}$ is a matrix formed by selecting 4 columns of identity matrix $\mathbf{I}_{N-2}$. Note that if $N=6$ then $\widehat{\mathbf{E}}=\mathbf{I}_{4}$ and $\mathbf{G}^{0}(\mathbf{x})=\widehat{\mathbf{G}}^{0}(\mathbf{x})$. In order to compute the desired solution of $\mathbf{G}^{1}(\mathbf{x})=\mathbf{0}$, where $\mathbf{G}^{1}(\mathbf{x})=\widehat{\mathbf{E}}^{T} \widehat{\mathbf{G}}^{1}(\mathbf{x})$, we consider the homotopy

$$
\mathbf{H}(\mathbf{x}, t)=\widehat{\mathbf{E}}^{T} \widehat{\mathbf{G}}(\mathbf{x}, t), \quad 0 \leqslant t \leqslant 1,
$$

where $\widehat{\mathbf{G}}(\mathbf{x}, t)$ is the system of equations of $\widehat{\mathbf{G}}(\mathbf{x})$ with parameter $\dot{r}_{j}=(1-$ $t) \dot{r}_{j}^{0}+t \dot{r}_{j}^{1}$. Then we follow the path of $\mathbf{H}(\mathbf{x}, t)=\mathbf{0}$ from $t=0$ to 1 with the starting point $\mathbf{x}_{0}$ by the continuation method.

\subsection{Algorithm 1 for $N=6$}

In this subsection we develop an algorithm for tracking the moving object with $N=6$ observation stations. Suppose that $\mathcal{C}_{0}=\{(\mathbf{x}(s), t(s)) \mid s \in \mathbb{R}\}$ is the solution path of $\mathbf{H}(\mathbf{x}, t)=\mathbf{0}$ with $(\mathbf{x}(0), t(0))=\left(\mathbf{x}_{0}, 0\right)$, where $\mathbf{H}(\mathbf{x}, t)$ is defined in (17) and $s$ is the arc length parameter. Next, we show that, generally, this path is regular, that is,

$$
\operatorname{rank}\left[\mathbf{H}_{\mathbf{x}}(\mathbf{x}(s), t(s)), \mathbf{H}_{t}(\mathbf{x}(s), t(s))\right]=4 \text { for } s \in \mathbb{R} .
$$

Remark 3.1. Suppose that $\mathcal{C}_{0}$ is regular. By Implicit Function Theorem we know that for each point $\left(\mathbf{x}\left(s_{0}\right), t\left(s_{0}\right)\right) \in \mathcal{C}_{0}$, there is a unique solution curve $\left\{(\mathbf{x}(s), t(s)) \mid s_{0}-\epsilon<s<s_{0}+\epsilon\right\}$ of $\mathbf{H}(\mathbf{x}, t)=\mathbf{0}$, where $\epsilon>0$. This means that there is no other solution path of $\mathbf{H}(\mathbf{x}, t)=\mathbf{0}$ which intersects with $\mathcal{C}_{0}$. The path $\mathcal{C}_{0}$ may have the fold bifurcation point (turning point) when $\mathbf{H}_{\mathbf{x}}(\mathbf{x}(s), t(s))$ is singular.

Now, we consider another homotopy

$$
\mathbf{H}_{\mathbf{F}} \equiv\left(H_{\mathbf{F}_{1}}, \ldots, H_{\mathbf{F}_{6}}\right): V \times[0,1] \rightarrow \mathbb{R}^{6},
$$


where $\mathbf{v}=(x, y, z, \dot{x}, \dot{y}, \dot{z})^{T}, V=\left\{\mathbb{R}^{3} \backslash \bigcup_{j=1}^{6}\left\{\mathbf{s}_{j}\right\}\right\} \times \mathbb{R}^{3}$ and

$$
H_{\mathbf{F}_{j}}(\mathbf{v}, t)=\frac{\left(x-x_{j}\right) \dot{x}+\left(y-y_{j}\right) \dot{y}+\left(z-z_{j}\right) \dot{z}}{\sqrt{\left(x-x_{j}\right)^{2}+\left(y-y_{j}\right)^{2}+\left(z-z_{j}\right)^{2}}}+t\left(\dot{r}_{j}^{0}-\dot{r}_{j}^{1}\right)-\dot{r}_{j}^{0} .
$$

Suppose that $t\left(\dot{r}_{j}^{0}-\dot{r}_{j}^{1}\right)-\dot{r}_{j}^{0} \neq 0$ for each $t \in[0,1]$ and $j=1, \ldots, 6$. From Remark 2.1, we know that

- if $\mathcal{C}_{\mathbf{F}}=\{(x(s), y(s), z(s), \dot{x}(s), \dot{y}(s), \dot{z}(s), t(s)) \mid s \in[0, \xi]\}$ is the solution path of $\mathbf{H}_{\mathbf{F}}(\mathbf{v}, t)=\mathbf{0}$ such that $\{t(s) \mid s \in[0, \xi]\} \subseteq[0,1]$, then $\mathcal{C}=\left\{\left(r_{3}(s), r_{4}(s), r_{5}(s), r_{6}(s), t(s)\right) \mid s \in[0, \xi]\right\}$ is a solution path of $\mathbf{H}(\mathbf{x}, t)=\mathbf{0}$, where

$$
r_{j}(s)=\sqrt{\left(x(s)-x_{j}\right)^{2}+\left(y(s)-y_{j}\right)^{2}+\left(z(s)-z_{j}\right)^{2}}, j=3,4,5,6 .
$$

- if $\mathcal{C}=\left\{\left(r_{3}(s), r_{4}(s), r_{5}(s), r_{6}(s), t(s)\right) \mid s \in[0, \xi]\right\}$ is a solution path of $\mathbf{H}(\mathbf{x}, t)=\mathbf{0}$ such that $\{t(s) \mid s \in[0, \xi]\} \subseteq[0,1]$, then

$$
\mathcal{C}_{\mathbf{F}}=\{(\mathbf{u}(s), \dot{\mathbf{u}}(s), t(s)) \mid s \in[0, \xi]\}
$$

is the solution path of $\mathbf{H}_{\mathbf{F}}(\mathbf{v}, t)=\mathbf{0}$, where $\mathbf{u}(s), \dot{\mathbf{u}}(s)$ can be obtained by $(7),(8)$ and (12).

Hence, $\mathcal{C}$ is regular if and only if $\mathcal{C}_{\mathbf{F}}$ is regular. Furthermore, $\mathcal{C}$ has a fold bifurcation point at $s=s_{0}$ if and only if $\mathcal{C}_{\mathbf{F}}$ has a fold bifurcation point at $s=s_{0}$. In the following, we show that each solution path of $\mathbf{H}_{\mathbf{F}}(\mathbf{v}, t)=\mathbf{0}$ is regular. To prove this, we need the sard's Theorem (see [9])

Theorem 3.2. [Sard's Theorem] Let $\mathbf{F}: U \rightarrow \mathbb{R}^{m}$ be a smooth function for some open set $U \subseteq \mathbb{R}^{n}$. Then the set

$$
\left\{\mathbf{F}(\mathbf{w}) \mid \mathbf{w} \in U \text { and } \operatorname{rank}\left(\mathbf{F}_{\mathbf{w}}(\mathbf{w})\right)<\min (m, n)\right\}
$$

is measure zero in $\mathbb{R}^{n}$.

In fact, the Doppler datas $\left\{\dot{r}_{j}\right\}_{j=1}^{6}$ contain measurement errors, i.e., $\dot{r}_{j}=\dot{r}_{j}^{e}+\epsilon$, where $\dot{r}_{j}^{e}$ is the exact value of the relative speed and $\epsilon$ is the measurement error which can be considered as a white noise. From Sard's Theorem, we have the following result.

Theorem 3.3. Suppose that $\left\{\dot{r}_{j}^{0}\right\}_{j=1}^{6}$ and $\left\{\dot{r}_{j}^{1}\right\}_{j=1}^{6}$ are measured Doppler datas at different times. Then each solution path of $\mathbf{H}_{\mathbf{F}}(\mathbf{v}, t)=\mathbf{0}$ is regular. 
Proof. Since $\left\{\dot{r}_{j}^{0}\right\}_{j=1}^{6}$ and $\left\{\dot{r}_{j}^{1}\right\}_{j=1}^{6}$ are measured Doppler datas, there are exact value of relative speeds $\dot{r}_{j}^{0 e}, \dot{r}_{j}^{1 e}$ and measurement errors $\epsilon_{j}^{0}, \epsilon_{j}^{1}$ such that

$$
\dot{r}_{j}^{0}=\dot{r}_{j}^{0 e}+\epsilon_{j}^{0} \text { and } \dot{r}_{j}^{1}=\dot{r}_{j}^{1 e}+\epsilon_{j}^{1} .
$$

Let $\epsilon_{j}=\epsilon_{j}^{0}-\epsilon_{j}^{1}$ for $j=1, \ldots, 6$. Then from (19), we have

$H_{\mathbf{F}_{j}}(\mathbf{v}, t)=\frac{\left(x-x_{j}\right) \dot{x}+\left(y-y_{j}\right) \dot{y}+\left(z-z_{j}\right) \dot{z}}{\sqrt{\left(x-x_{j}\right)^{2}+\left(y-y_{j}\right)^{2}+\left(z-z_{j}\right)^{2}}}+t\left(\dot{r}_{j}^{0 e}-\dot{r}_{j}^{l e}+\epsilon_{j}\right)-\dot{r}_{j}^{0 e}-\epsilon_{j}^{0}$.

Suppose that $\dot{r}_{j}^{0}-\dot{r}_{j}^{1}=\dot{r}_{j}^{0 e}-\dot{r}_{j}^{1 e}+\epsilon_{j}$ for $j=1, \ldots, 6$ are given and fixed. Then the measurement error $\epsilon_{j}^{0}$ can be considered as a random number. It follows from Sard's Theorem that each solution path of $\mathbf{H}_{\mathbf{F}}(\mathbf{v}, t)=\mathbf{0}$ is regular.

Assume that $t\left(\dot{r}_{j}^{0}-\dot{r}_{j}^{1}\right)-\dot{r}_{j}^{0} \neq 0$ for each $t \in[0,1]$ and $j=1, \ldots, 6$. Let $\left(\mathbf{x}_{0}, 0\right)$ be a real solution of $\mathbf{H}(\mathbf{x}, t)=\mathbf{0}$, where $\mathbf{H}(\mathbf{x}, t)$ is defined in (17). Suppose that $\mathcal{C}_{0}=\{(\mathbf{x}(s), t(s)) \mid s \in[0, \xi]\}$ is a real solution path of $\mathbf{H}(\mathbf{x}, t)=\mathbf{0}$ with $(\mathbf{x}(0), t(0))=\left(\mathbf{x}_{0}, 0\right)$, where $\{t(s) \mid s \in[0, \xi]\} \subseteq[0,1], \xi>0$ and $t(\xi) \in\{0,1\}$. From Theorem 3.3 we know that the solution path $\mathcal{C}_{0}$ is regular. Suppose that

- there is no fold bifurcation point on $\mathcal{C}_{0}$ :

We can employ the homotopy continuation method (see [5]) to follow the path $\mathcal{C}_{0}$ in the real variable $t \in[0,1]$. In this case, it holds that $t(\xi)=1$. Hence, the solution, $\mathbf{x}_{1}=\mathbf{x}(\xi)$, of $\mathbf{H}(\mathbf{x}, 1)=\mathbf{0}$ can be achieved.

- $\mathcal{C}_{0}$ contains fold bifurcations:

Suppose that $\left(\mathbf{x}^{*}, t^{*}\right) \in \mathcal{C}_{0}$ is a fold bifurcation point on $\mathcal{C}_{0}$. In $[6,4]$, it shows that there are two branches of solution paths, $\mathcal{C}_{0}$ and $\mathcal{C}_{0}^{c}$, passing through $\left(\mathbf{x}^{*}, t^{*}\right)$, where $\mathcal{C}_{0}^{c}$ is complex curve. If $\phi \in \mathbb{R}^{5}$ is the tangent vector of the path of $\mathcal{C}_{0}$ at $\left(\mathbf{x}^{*}, t^{*}\right)$, then the tangent vector of $\mathcal{C}_{0}^{c}$ is the direction of $i \phi$ (see Figure 1). To compute a solution $\mathbf{x}_{1}$ of $\mathbf{H}(\mathbf{x}, 1)=\mathbf{0}$ which connects with the starting point $\mathbf{x}_{0}$, we need to follow the complex bifurcation branch $\mathcal{C}_{0}^{c}$ from the fold bifurcation point $\left(\mathbf{x}^{*}, t^{*}\right)$. Since the coefficients of $\mathbf{H}(\mathbf{x}, t)$ are real, the path $\mathcal{C}_{0}^{c}$ consists of a complex path and its complex conjugacy, hence, we only need to follow one of them with tangent vector $i \phi$ or $-i \phi$. Note that the computed solution $\mathbf{x}_{1}$ may be complex if those two paths of $\mathcal{C}_{0}^{c}$ (the complex path and its complex conjugacy) do not intersect before $t$ goes to 1 . 


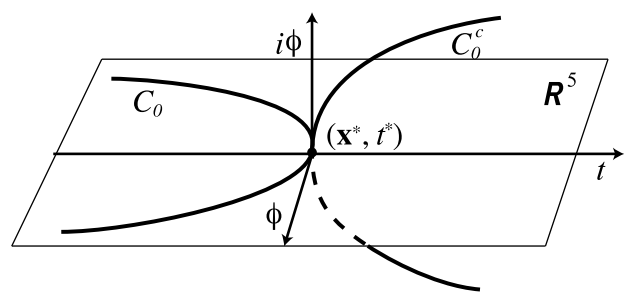

Figure 1: Two solution paths $\mathcal{C}_{0}$ and $\mathcal{C}_{0}^{c}$.

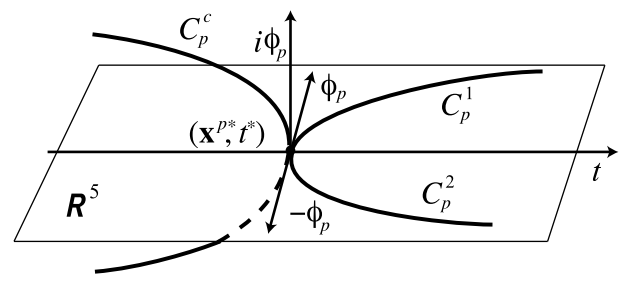

Figure 2: Two solution paths $\mathcal{C}_{p}^{c}$ and $\mathcal{C}_{p}=\mathcal{C}_{p}^{1} \cup \mathcal{C}_{p}^{2}$.

Remark 3.4. If the real path $\mathcal{C}_{0}$ contains a fold bifurcation point, then the computed solution $\mathbf{x}_{1}$ of $\mathbf{H}(\mathbf{x}, 1)=\mathbf{0}$ may be complex. Since the solution $\mathbf{x}_{1}$ describes the distances between the moving object and stations, $\mathbf{x}_{1}$ should be real and positive. So, if the starting point $\mathbf{x}_{0}$ of $\mathcal{C}_{0}$ represents the distances between the moving object and stations, then the computed solution $\mathbf{x}_{1}$ is complex because of measurement errors in measured datas. Intuitively, the complex path will bifurcate and produce two real paths when we follow the path of the homotopy $\mathbf{H}^{k}(\mathbf{x}, t)$, where $\mathbf{H}^{k}(\mathbf{x}, t)$ is formed by $\widehat{\mathbf{G}}(\mathbf{x})$ in (16) with $\dot{r}_{j}=(1-t) \dot{r}_{j}^{k}+t \dot{r}_{j}^{k+1},\left\{\dot{r}_{j}^{k}\right\}_{j=1}^{6}$ and $\left\{\dot{r}_{j}^{k+1}\right\}_{j=1}^{6}$ are measured datas at times $t_{k}$ and $t_{k+1}$, respectively.

Let $\mathcal{C}_{k}^{c}=\left\{\left(\mathbf{x}^{k}(t), t\right) \mid 0 \leqslant t \leqslant 1\right\}$ be the complex solution path of $\mathbf{H}^{k}(\mathbf{x}, t)=\mathbf{0}$ for $k=1, \ldots, p-1$ such that $\mathbf{x}^{1}(0)=\mathbf{x}_{1}$ and $\mathbf{x}^{k}(0)=\mathbf{x}^{k-1}(1)$ for $k>1$. Suppose that the complex branch, $\mathcal{C}_{p}^{c}=\left\{\left(\mathbf{x}^{p}(s), t(s)\right) \mid s \in\left[0, \xi_{p}\right]\right\}$ with $\mathbf{x}^{p}(0)=\mathbf{x}^{p-1}(1)$, contains a real fold bifurcation point $\left(\mathbf{x}^{p *}, t^{*}\right) \equiv$ $\left(\mathbf{x}^{p}\left(s^{*}\right), t\left(s^{*}\right)\right) \in \mathbb{R}^{5}$. If $\varphi_{p}=i \phi_{p}\left(\phi_{p} \in \mathbb{R}^{5}\right)$ is the tangent vector of the path of $\mathcal{C}_{p}^{c}$ at $\left(\mathbf{x}^{p *}, t^{*}\right)$, then the tangent vector of the real bifurcation branch $\mathcal{C}_{p}=\mathcal{C}_{p}^{1} \cup \mathcal{C}_{p}^{2}$ is the direction of $\phi_{p}$ (see Figure 2). Because we do not know which one of bifurcation paths, $\mathcal{C}_{p}^{1}$ or $\mathcal{C}_{p}^{2}$, can be used to trace the moving object, hence, we need to follow both of them.

Remark 3.5. Due to the measurement errors of the measured data, if a complex solution $\mathbf{x}=\mathbf{x}_{R}+i \mathbf{x}_{I}$ is obtained when we trace the solution path 
of a homotopy, then we set $\left(r_{3}, r_{4}, r_{5}, r_{6}\right)=\mathbf{x}_{R}$ and compute the position $\mathbf{u}$ and velocity $\dot{\mathbf{u}}$ of the moving object by (7), (8) and (12).

Given $N=6$ stations $\left\{\mathbf{s}_{j}\right\}_{j=1}^{6}$ such that the first $N-4$ rows of the matrix $\widehat{\mathbf{A}}$ defined in (11) are linearly independent. Suppose that $\left\{\dot{r}_{j}^{k}\right\}_{j=1}^{6}$ for $k=$ $0,1, \ldots, m$ are measured Doppler datas at times $t_{0}, t_{1}, \ldots, t_{m}$, respectively. Let $\mathbf{H}^{k}(\mathbf{x}, t)$ for $k=0, \ldots, m-1$ be the homotopy which formed by $\widehat{\mathbf{G}}(\mathbf{x})$ in (16) with $\dot{r}_{j}=(1-t) \dot{r}_{j}^{k}+t \dot{r}_{j}^{k+1}$. Suppose that $\mathbf{x}_{0}=\left(r_{3}^{0}, r_{4}^{0}, r_{5}^{0}, r_{6}^{0}\right)^{T} \in \mathbb{R}^{4}$ is a solution of $\mathbf{H}^{0}(\mathbf{x}, 0)=\mathbf{0}$, where $r_{j}^{0}$ describes the distance between the moving object and the station $\mathbf{s}_{j}$ at time $t_{0}$. We design an efficient procedure for tracking the moving object at times $t_{0}, t_{1}, \ldots, t_{m}$. The flowchart of Algorithm 1 is shown in Figure 3.

\subsection{Algorithm 2 for $N>6$}

When $N>6, \widehat{\mathbf{G}}(\mathbf{x})=\mathbf{0}$ in (16) is an overdetermined system. In this subsection, we propose a method to choose a suitable subsystem $\mathbf{G}^{\star}(\mathbf{x})=\mathbf{0}$ of $\widehat{\mathbf{G}}(\mathbf{x})=\mathbf{0}$ and compute its desired solution. Suppose that we have a subsystem $\mathbf{G}(\mathbf{x})=\mathbf{0}$ with the desired solution $\mathbf{x}_{0}$. Intuitively, a suitable subsystem $\mathbf{G}^{\star}(\mathbf{x})=\mathbf{0}$ should have a good condition number of Jacobian matrix $\mathbf{G}_{\mathbf{x}}^{\star}\left(\mathbf{x}_{0}\right) \in \mathbb{R}^{4 \times 4}$. Let $\widehat{\mathbf{G}}_{\mathbf{x}}\left(\mathbf{x}_{0}\right) \in \mathbb{R}^{(N-2) \times 4}$ be the Jacobian matrix of $\widehat{\mathbf{G}}(\mathbf{x})=\mathbf{0}$ at $\mathbf{x}=\mathbf{x}_{0}$. Then $\mathbf{G}_{\mathbf{x}}^{\star}\left(\mathbf{x}_{0}\right)$ is a submatrix of $\widehat{\mathbf{G}}_{\mathbf{x}}\left(\mathbf{x}_{0}\right)$. Let

$$
\widehat{\mathbf{G}}_{\mathbf{x}}\left(\mathbf{x}_{0}\right)^{T} \mathbf{E}=\mathbf{Q R}
$$

be the QR factorization with column pivoting of $\widehat{\mathbf{G}}_{\mathbf{x}}\left(\mathbf{x}_{0}\right)^{T}$, where $\mathbf{E}$ is a permutation matrix. The suitable system $\mathbf{G}^{\star}(\mathbf{x})$ can be easily chosen by

$$
\mathbf{G}^{\star}(\mathbf{x})=\widehat{\mathbf{E}}^{T} \widehat{\mathbf{G}},
$$

where $\widehat{\mathbf{E}}=\mathbf{E}(:, 1: 4)$. If $\mathbf{G}^{\star}(\mathbf{x}) \neq \mathbf{G}(\mathbf{x})$, then we can follow the path of the homotopy

$$
\mathbf{H}(\mathbf{x}, t)=(1-t) \mathbf{G}(\mathbf{x})+t \mathbf{G}^{\star}(\mathbf{x})
$$

from $t=0$ to 1 with the starting point $\mathbf{x}_{0}$ to compute a solution $\mathbf{x}_{*}$ of $\mathbf{G}^{\star}(\mathbf{x})=\mathbf{0}$.

Remark 3.6. Let $N>6$ and $\left\{\dot{r}_{j}\right\}_{j=1}^{N}$ be the speeds of the moving object with respect to the observation stations. Then the overdetermined system $\mathbf{F}(\mathbf{v})=\mathbf{0}$ has exactly one solution, where $\mathbf{F}=\left(F_{1}, \ldots, F_{N}\right)$ is given in (3). 


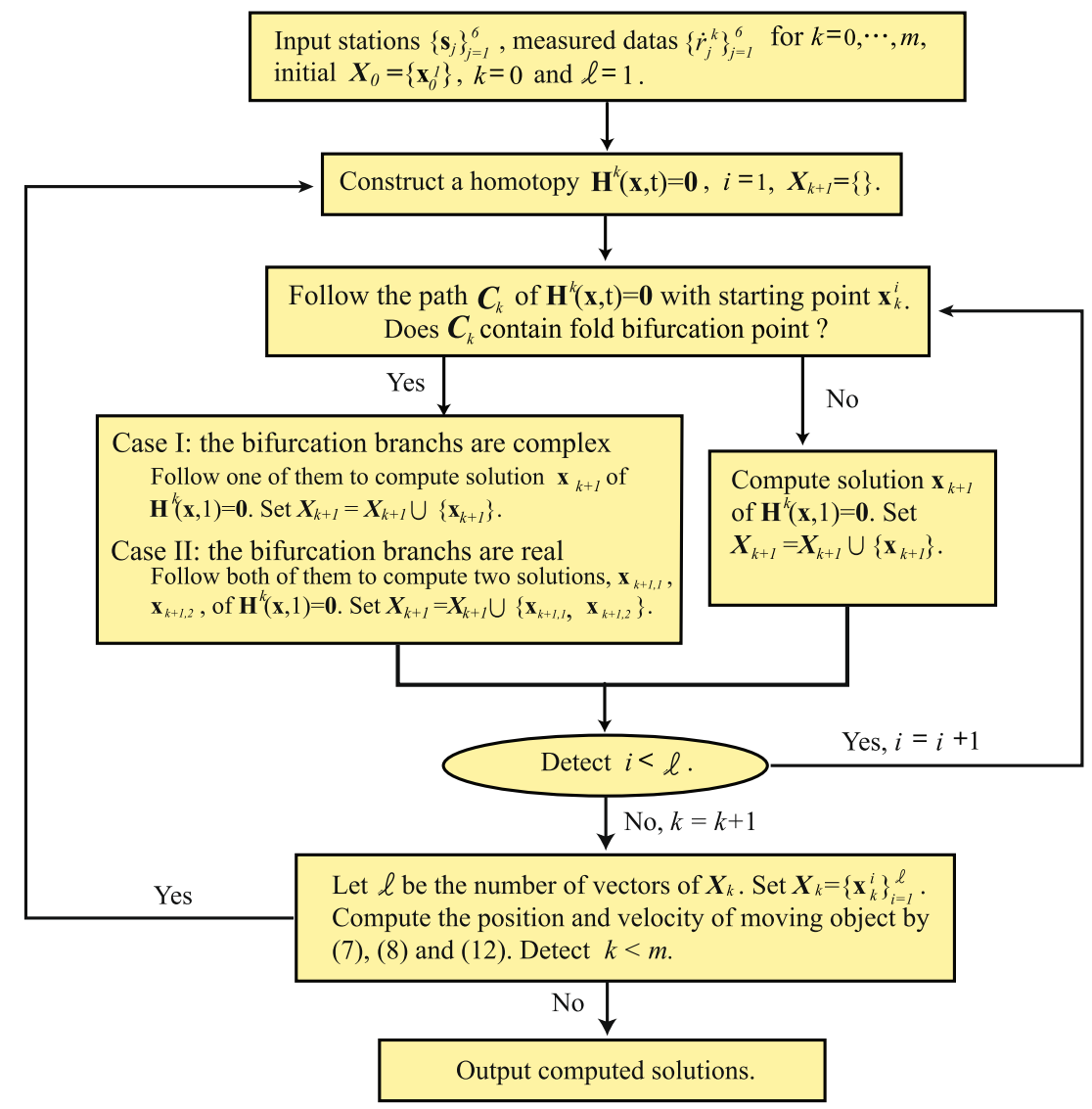

Figure 3: The flowchart of Algorithm 1 for the Doppler tracking procedure with $N=6$.

Then it is easily seen that $\widehat{\mathbf{G}}(\mathbf{x})=\mathbf{0}$ in (16) has exactly one solution $\mathbf{x}_{0}$ by Remark 2.1. Since $\mathbf{G}(\mathbf{x})$ and $\mathbf{G}^{\star}(\mathbf{x})$ are subsystems of $\widehat{\mathbf{G}}(\mathbf{x}),\left\{\left(\mathbf{x}_{0}, t\right) \mid 0 \leqslant\right.$ $t \leqslant 1\}$ is the solution path of the homotopy in (20), hence $\mathbf{x}_{*}=\mathbf{x}_{0}$ and $\mathbf{x}_{*}$ is also a desired solution of $\mathbf{G}^{\star}(\mathbf{x})=\mathbf{0}$. If those $N$ measured Doppler datas $\left\{\dot{r}_{j}\right\}_{j=1}^{N}$ are perturbed and $\mathbf{x}_{0}$ is the desired solution of $\mathbf{G}(\mathbf{x})=\mathbf{0}$, then the terminating point, $\mathbf{x}_{*}$, of the solution path of the homotopy in (20) will approximate $\mathbf{x}_{0}\left(\mathbf{x}_{*} \approx \mathbf{x}_{0}\right)$ and is also a desired solution of $\mathbf{G}^{\star}(\mathbf{x})=\mathbf{0}$.

Assume that $\left\{\dot{r}_{j}^{0}\right\}_{j=1}^{N}$ and $\left\{\dot{r}_{j}^{1}\right\}_{j=1}^{N}$ are measured speeds of a moving object by Doppler effect from those $N>6$ stations at time $t_{0}$ and $t_{1}$, respectively, where $t_{0} \simeq t_{1}$. Let $\widehat{\mathbf{G}}^{0}(\mathbf{x})$ and $\widehat{\mathbf{G}}^{1}(\mathbf{x})$ be given in (16) with parameters $\dot{r}_{j}=\dot{r}_{j}^{0}$ and $\dot{r}_{j}=\dot{r}_{j}^{1}$, respectively. Suppose that $\mathbf{G}^{0}(\mathbf{x})=\widehat{\mathbf{E}}^{T} \widehat{\mathbf{G}}^{0}(\mathbf{x})$ is the 


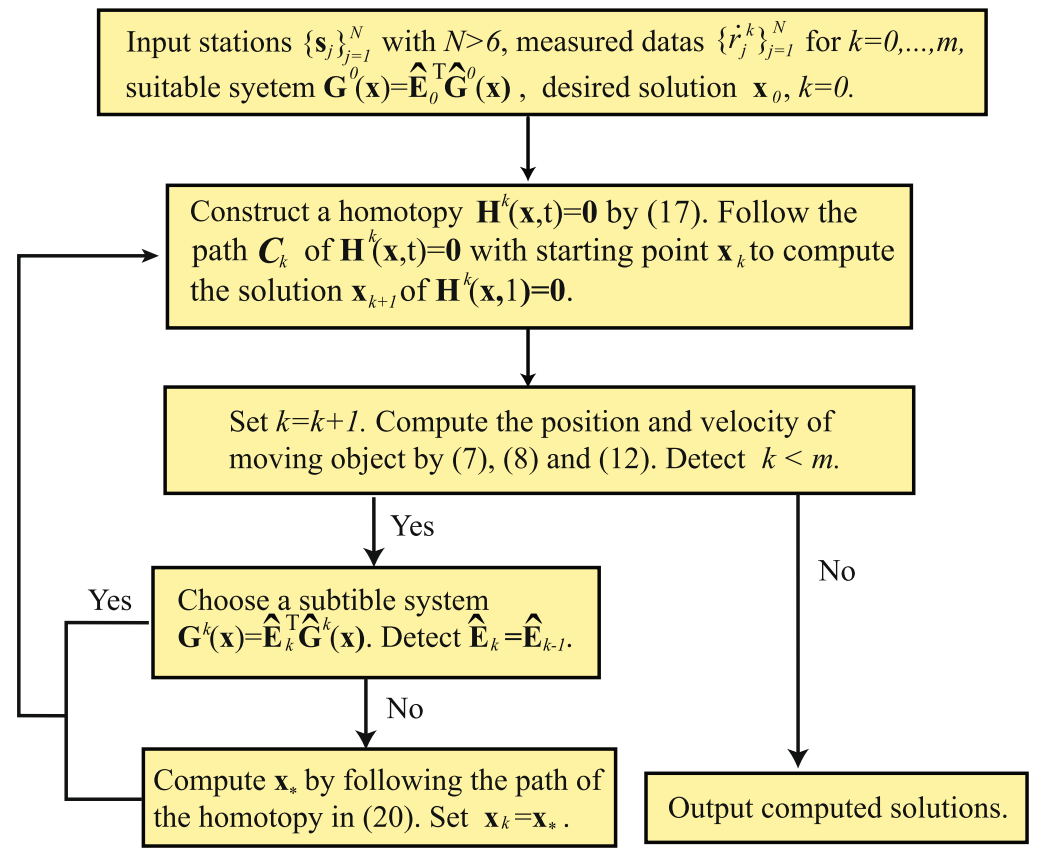

Figure 4: The flowchart of Algorithm 2 for the Doppler tracking procedure with $N>6$.

suitable subsystem and $\mathbf{x}_{0}$ is the desired solution of $\mathbf{G}^{0}(\mathbf{x})=\mathbf{0}$. Then the solution path with the starting point $\mathbf{x}_{0}$ for the homotopy, $\mathbf{H}(\mathbf{x}, t)=\mathbf{0}$ defined in (17), always has no fold bifurcation. We design an efficient procedure for tracking the moving object at times $t_{0}, t_{1}, \ldots, t_{m}$ when $N>6$ and the flowchart of Algorithm 2 is shown in Figure 4.

\section{Numerical experiments}

In this section we will show some numerical results of tracking a moving object. Given $N=6$ observation stations located at

$$
\begin{array}{ll}
\mathbf{s}_{1}=10^{3} \times(449,-17,-122)^{T} & \mathbf{s}_{2}=10^{3} \times(24,-13,-384)^{T} \\
\mathbf{s}_{3}=10^{3} \times(181,-5,166)^{T} & \mathbf{s}_{4}=10^{3} \times(396,-14,142)^{T} \\
\mathbf{s}_{5}=10^{3} \times(51,-4,209)^{T} & \mathbf{s}_{6}=10^{3} \times(147,-3,-89)^{T} .
\end{array}
$$

We take the real trajectory of a moving object and the associated velocity from $t=0$ to $t=110$ (seconds) provided by [11] and shown in Figure 5 . We divide the time interval into $m=2200$ subintervals using 2201 equally 
(a) The real position of the moving object

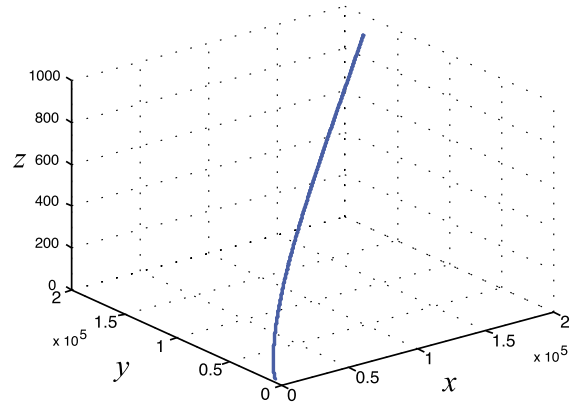

(b) The real velocity of the moving object

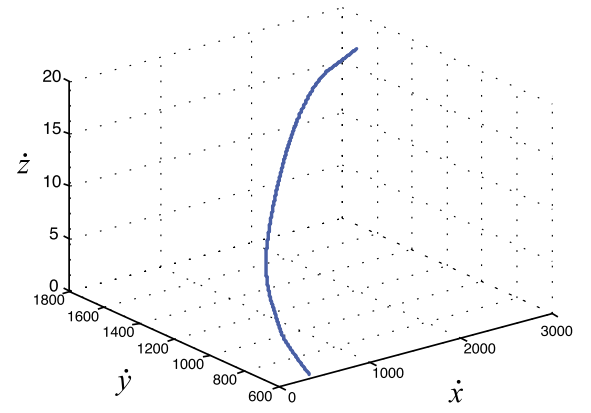

Figure 5: The real position and the real velocity of the moving object.

spaced nodes $t_{k}=0.05 \times k, k=0, \ldots, m$. Let $\mathbf{u}_{k}^{e}=\left(x\left(t_{k}\right), y\left(t_{k}\right), z\left(t_{k}\right)\right)^{T}$ and $\dot{\mathbf{u}}_{k}^{e}=\left(\dot{x}\left(t_{k}\right), \dot{y}\left(t_{k}\right), \dot{z}\left(t_{k}\right)\right)^{T}$, be the real position and the real velocity of the moving object at time $t_{k}$. Then the speeds of the moving object with respect to the stations $\mathbf{s}_{j}$ at time $t_{k}$ can be computed by

$$
\dot{r}_{j}^{e, k}=\frac{\left(\mathbf{u}_{k}^{e}-\mathbf{s}_{j}\right)^{T} \dot{\mathbf{u}}_{k}^{e}}{\left\|\mathbf{u}_{k}^{e}-\mathbf{s}_{j}\right\|}, \quad j=1, \ldots, 6 .
$$

Let $\mathbf{F}: \mathbb{R}^{6} \rightarrow \mathbb{R}^{N}$ be given in (3). The degree of $\mathbf{F}$ at $\mathbf{v}_{k}^{e}=\left(\mathbf{u}_{k}^{e^{T}}, \dot{\mathbf{u}}_{k}^{e^{T}}\right)^{T}$ is defined as

$$
\operatorname{deg}\left(\mathbf{F}, \mathbf{v}_{k}^{e}\right)=\operatorname{Sgn}\left(D_{\mathbf{v}} \mathbf{F}\left(\mathbf{v}_{k}^{e}\right)\right)
$$

where $D_{\mathbf{v}} \mathbf{F}\left(\mathbf{v}_{k}^{e}\right) \in \mathbb{R}^{6 \times 6}$ is the Jacobian matrix of $\mathbf{F}(\mathbf{v})=\mathbf{0}$ at $\mathbf{v}=\mathbf{v}_{k}^{e}$. The degrees of $\mathbf{F}$, for $k=0,1, \ldots, m$, are shown in Figure 6 . We see that the degree changes from 1 to -1 when $k=k^{*}=1184$ (i.e., $\operatorname{deg}\left(\mathbf{F}, \mathbf{v}_{k^{*}}^{e}\right)=1$ and $\left.\operatorname{deg}\left(\mathbf{F}, \mathbf{v}_{k^{*}+1}^{e}\right)=-1\right)$. It follows from Bifurcation Test Theorem (see [5]) that the solution path of $\mathbf{H}_{F}^{k^{*}}(\mathbf{v}, t)=\mathbf{0}$ with the starting point $\mathbf{v}_{k^{*}}^{e}$ has a bifurcation point (transcritical bifurcation point), where the homotopy $\mathbf{H}_{F}^{k^{*}}(\mathbf{v}, t)$ is defined in (18) in which $\dot{r}_{j}^{0}$ and $\dot{r}_{j}^{1}$ are replaced by $\dot{r}_{j}^{e, k^{*}}$ and $\dot{r}_{j}^{e, k^{*}+1}$, respectively.

In the homotopy continuation procedure, it needs to trace a solution path of the homotopy $\mathbf{H}^{k}(\mathbf{x}, t)=\mathbf{0}$ from $t=0$ to $t=1$ by the continuation method for each $k=0,1, \ldots, m-1$. Continuation method mainly consists of the prediction scheme and the correction scheme. In prediction scheme, the predictor can be obtained by solving a linear system with an adaptive timestep. In correction scheme, we employ Newton method to compute a new 


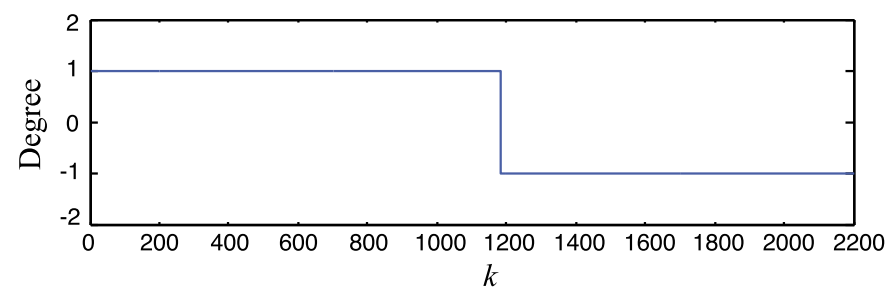

Figure 6: The degree, $\operatorname{deg}\left(\mathbf{F}, \mathbf{v}_{k}^{e}\right)$, for $k=0,1, \ldots, m$.

(a) Relative errors of positions

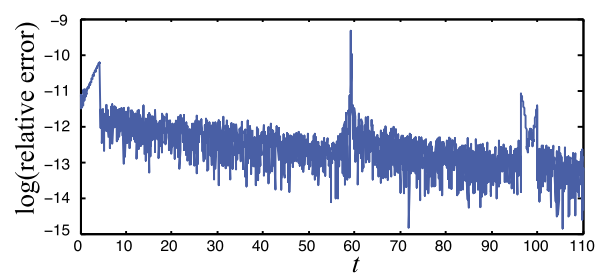

(b) Relative errors of velocities

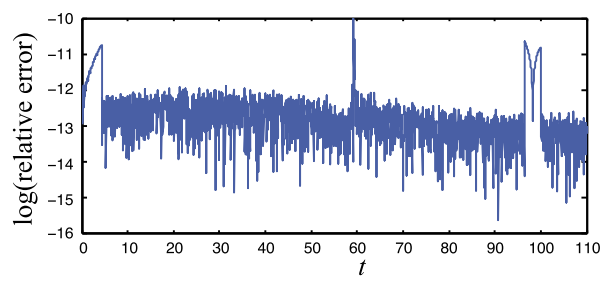

Figure 7: The relative errors potted by the log scale.

approximation to the solution path. Hence, for tracking the solution path, it needs to solve a series of linear systems. In the following experiments, we will show the number of linear systems which need to be solved while the solution path of the homotopy $\mathbf{H}^{k}(\mathbf{x}, t)=\mathbf{0}$ is followed.

Experiment 1: In this experiment we show the numerical results with the measured data having no measurement errors (i.e., $\dot{r}_{j}^{k}=\dot{r}_{j}^{e, k}, j=1, \ldots, 6$, $k=0,1, \ldots, m)$. We use Algorithm 1 (see flowchart in Figure 3) to trace the moving object time by time. The relative errors for positions and velocities plotted by the log scale are shown in Figure $7(a)$ and $(b)$, respectively. We see that the average of relative errors is close to $10^{-13}$ which is satisfactory in numerical computation. Figure 8 shows that the numbers of solving linear systems are between $2 \sim 4$ while the solution path of $\mathbf{H}^{k}(\mathbf{x}, k)=\mathbf{0}$ is followed from $t=0$ to $t=1$ with various time-steps: $k=0,1, \ldots, m-1$. Algorithm 1 is efficient and reliable for this case because for almost $99 \%$ of time-steps $k$, it only needs to solve 3 linear systems to get the next solution $\mathbf{x}_{k+1}$ of $\mathbf{H}^{k}(\mathbf{x}, 1)=\mathbf{0}$.

Experiment 2: In this experiment, we show the numerical results with measured data by Doppler effect having measurement errors (i.e., $\dot{r}_{j}^{k}=$ $\dot{r}_{j}^{e, k}+\epsilon_{j}^{k}$, where $\epsilon_{j}^{k}$ is the measurement error provided by $[11], j=1, \ldots, 6$, 


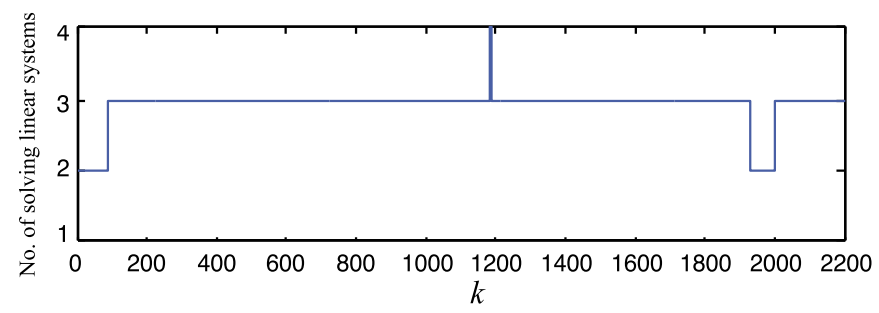

Figure 8: The number of solving linear systems while we follow the solution path of $\mathbf{H}^{k}(\mathbf{x}, t)=\mathbf{0}$ from $t=0$ to $t=1$ for $k=0,1, \ldots, m-1$.

(a) The position of the computed orbit $\left(\mathcal{P}_{1}\right)$

(b) The position of the computed orbit $\left(\mathcal{P}_{2}\right)$
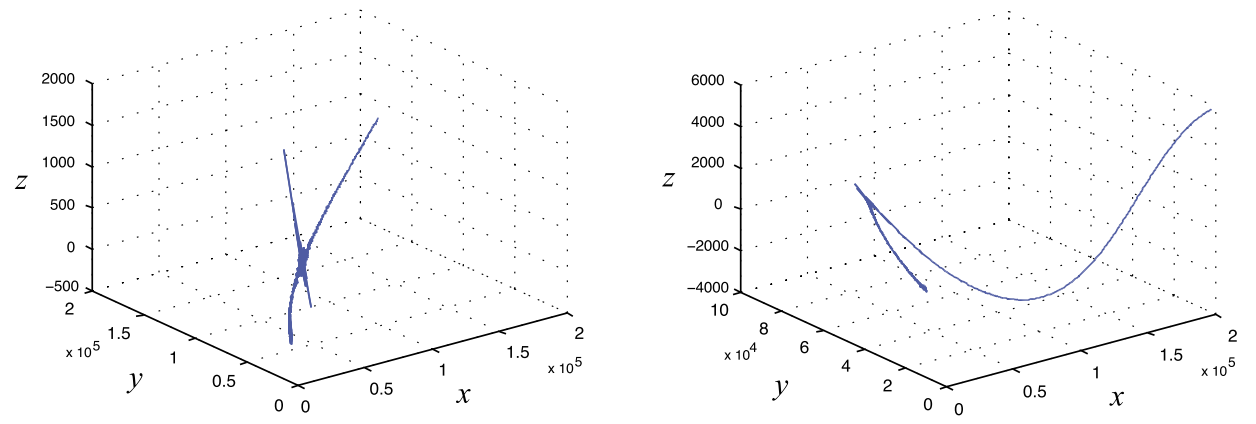

Figure 9: The positions of two computed orbits, $\mathcal{P}_{1}$ and $\mathcal{P}_{2}$.

$k=0,1, \ldots, m)$. As above, using Algorithm 1 (in Figure 3) to trace the trajectory of the moving object, it will meet six times at fold bifurcation points (turing points) on the path $\mathcal{C}_{k}: \mathbf{H}^{k}(\mathbf{x}, t)=\mathbf{0}$ for $k=1174,1177$, $1181,1190,1192,1194$, caused by the measurement errors by Doppler effect. More precisely, the solution paths bifurcate into two complex conjugate paths for $k$ in the union of intervals: $I_{c} \equiv(1174,1177) \cup(1181,1190) \cup$ $(1192,1194)$, and bifurcate into two real paths for $k$ in the union of intervals: $I_{2 p} \equiv[1177,1181] \cup[1190,1192] \cup[1194,2200]$. We denote two computed trajectories of the moving object by $\mathcal{P}_{1}$ and $\mathcal{P}_{2}$, respectively. Note that $\mathcal{P}_{1}=\mathcal{P}_{2}$ for $k \in[0,1174] \cup I_{c}$ and $\mathcal{P}_{1} \neq \mathcal{P}_{2}$ for $k \in I_{2 p}$. In Figure 9 we show the positions of computed orbits $\mathcal{P}_{1}$ and $\mathcal{P}_{2}$ for the moving object. In Figure 10 we show the associated relative errors plotted by the $\log$ scale. The computed orbit $\mathcal{P}_{1}$ oscillates and has large relative error (about $10^{-1}$ ) near $t_{k^{*}}=0.05 \times k^{*}=59.2$. The position of computed orbit $\mathcal{P}_{1}$ projected onto the $x, y$ and $z$ coordinates are shown in Figure 11 (a), (b) and (c), respectively. We see that the $z$-coordinate of the orbit $\mathcal{P}_{1}$ 
(a) Relative errors of $\mathcal{P}_{1}$

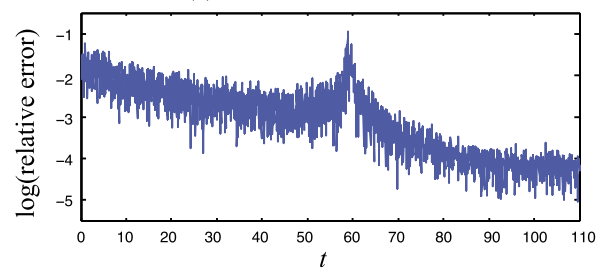

(b) Relative errors of $\mathcal{P}_{2}$

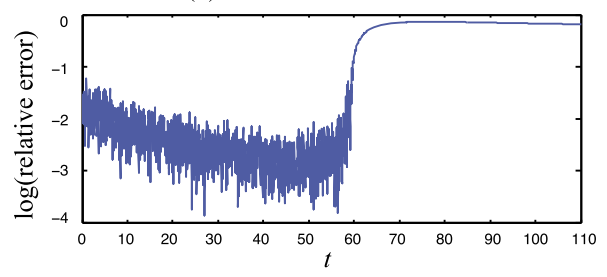

Figure 10: The relative errors of two computed orbits, $\mathcal{P}_{1}$ and $\mathcal{P}_{2}$, plotted by the $\log$ scale.

(a) $x$-coordinate of $\mathcal{P}_{1}$

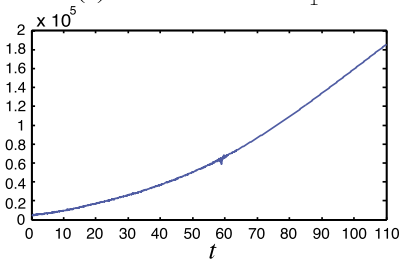

(b) $y$-coordinate of $\mathcal{P}_{1}$

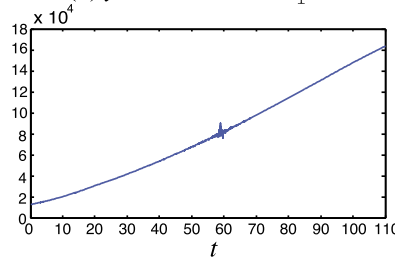

(c) z-coordinate of $\mathcal{P}_{1}$

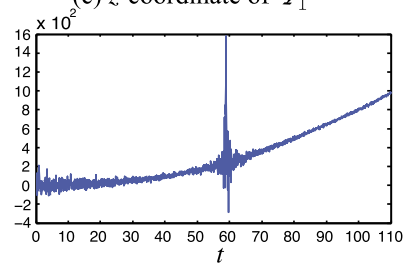

Figure 11: The position of the computed orbit $\left(\mathcal{P}_{1}\right)$ projected onto the $x, y$ and $z$ coordinates.

oscillates between $1.6 \times 10^{3}$ and $-3 \times 10^{2}$ as $t$ approaches to 59.2. When $t>65$, the relative error of $\mathcal{P}_{1}$ is less than $10^{-3}$. Thus, the orbit $\mathcal{P}_{1}$ really tracks the positions of the moving object. By Bifurcation Test Theorem, in this example, we know that there is another path intersects with the orbit of moving object. The orbit $\mathcal{P}_{2}$ for $t>59.2$ is just the path which intersects with the orbit of the moving object and is redundant in the tracking process. Note that the relative errors of $\mathcal{P}_{2}$ are large (about $\left.10^{0}\right)$ for $t>65$. In Figure 12 we show that the number of solving linear systems of Algorithm 1 while we follow the solution path of $\mathbf{H}^{k}(\mathbf{x}, t)=\mathbf{0}$ from $t=0$ to $t=1$ with various time-steps: $k=0,1, \ldots, m-1$. We see that the continuation method needs to solve about 100 linear systems to get the solution for the next step when $k$ approaches to $k^{*}=1184$. When $k<1174$ or $k>1194$, the number of solving linear systems is less than 5 .

In order to report the numerical results by Algorithm 2 with $N>6$, we add an additional station located at $\mathbf{s}_{7}=(0,0,0)^{T}$. The speed of the moving object with respect to the station $\mathbf{s}_{7}$ at time $t_{k}$ measured by Doppler effect can be constructed by 


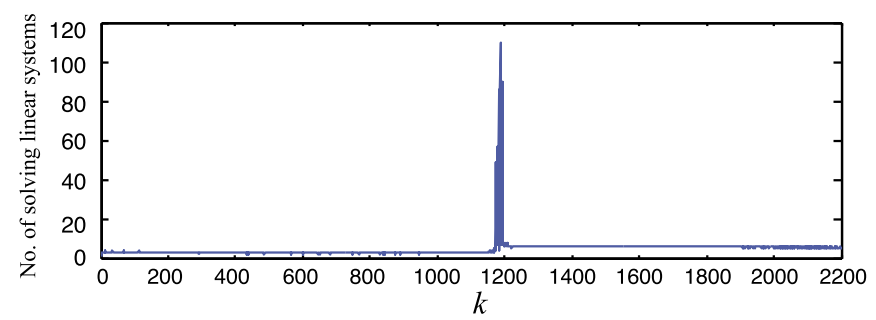

Figure 12: The number of solving linear systems while we follow the solution path of $\mathbf{H}^{k}(\mathbf{x}, t)=\mathbf{0}$ from $t=0$ to $t=1$ for $k=0,1, \ldots, m-1$.

(a) The position of the computed orbit

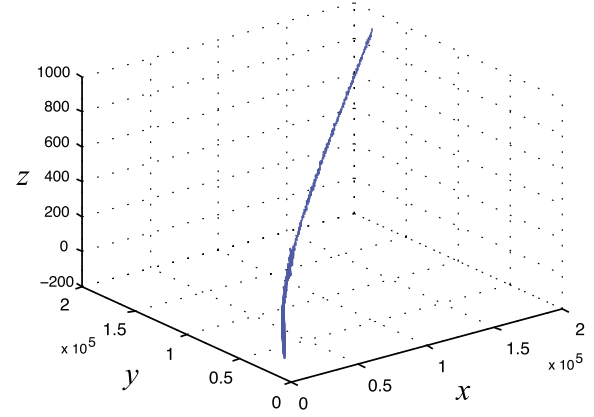

(b) The velocity of the computed orbit

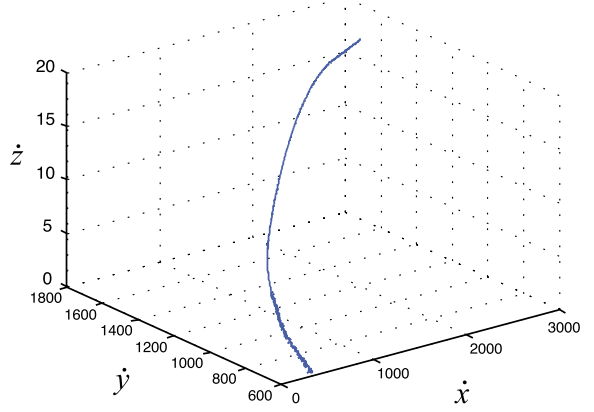

Figure 13: The position and the velocity of the computed orbit.

$$
\dot{r}_{7}^{k}=\frac{\left(\mathbf{u}_{k}^{e}-\mathbf{s}_{7}\right)^{T} \dot{\mathbf{u}}_{k}^{e}}{\left\|\mathbf{u}_{k}^{e}-\mathbf{s}_{7}\right\|}+\epsilon_{7}^{k}, \quad k=0,1, \ldots, m
$$

where the errors $\epsilon_{7}^{k}$ are randomly generated with $\left|\epsilon_{7}^{k}\right| \approx 0.01 \times\left|\frac{\left(\mathbf{u}_{k}^{e}-\mathbf{s}_{7}\right)^{T} \dot{\mathbf{u}}_{k}^{e}}{\left\|\mathbf{u}_{k}^{e}-\mathbf{s}_{7}\right\|}\right|$.

Experiment 3: In this experiment we show the numerical results by Algorithm 2 (see flowchart in Figure 4 with $N=7$ ) with the measured data by Doppler effect having measurement errors (i.e., $\dot{r}_{j}^{k}=\dot{r}_{j}^{e, k}+\epsilon_{j}^{k}$, where $\epsilon_{j}^{k}$ is the measured error, $\left.j=1, \ldots, 7, k=0,1, \ldots, m\right)$. In Figure 13 , we show the position and the velocity of the computed orbit for the moving object which are close to the position and the velocity of the real moving object in Figure 5. The relative errors in Figure 14 plotted by the $\log$ scale are between $10^{-3} \sim 10^{-5}$ which are satisfactory. In Figure 15, we show the numbers of solving linear systems while we follow the solution path of $\mathbf{H}^{k}(\mathbf{x}, t)=\mathbf{0}$ from $t=0$ to $t=1$ with various time-steps: $k=0,1, \ldots, m-1$. We see that the maximal number is 7 and $99 \%$ of 
(a) Relative errors of positions

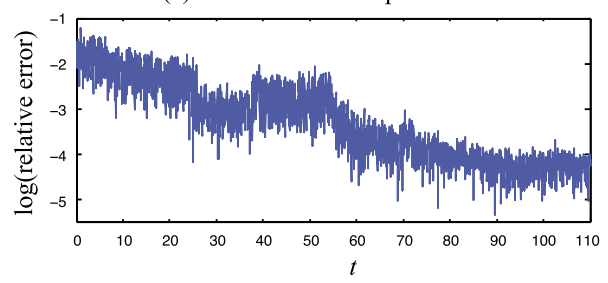

(b) Relative errors of velocities

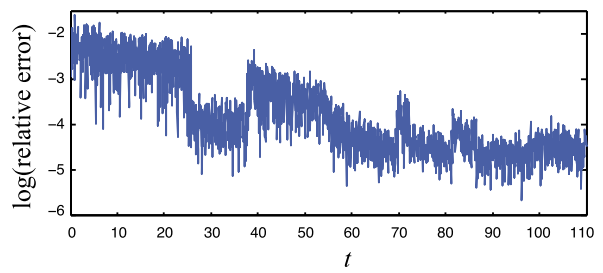

Figure 14: The relative errors potted by the log scale.

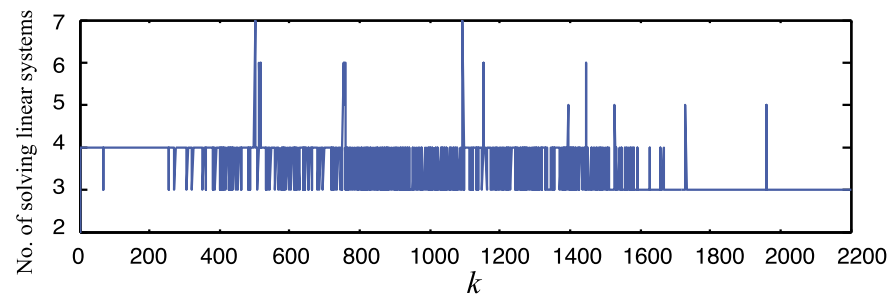

Figure 15: The number of solving linear systems while we follow the solution path of $\mathbf{H}^{k}(\mathbf{x}, t)=\mathbf{0}$ from $t=0$ to $t=1$ for $k=0,1, \ldots, m-1$.

numbers is between $3 \sim 4$. Thus, the Algorithm 2 is quite promising and robust.

\section{Conclusions}

In this paper, we propose a novel simplification to reduce the original system of a moving object to a system of $N-2$ polynomials in 4 unknowns, where $N$ is the number of observation stations. Using the simplified systems with different measured Doppler data, we constructed a series of homotopy functions. Then the position and the velocity of the moving object can be obtained by following the solution path of those homotopy functions. When $N=6$ we prove that, generically, each solution path of the homotopy is regular. In this case, we need to deal with the fold bifurcation when we trace the solution path. When $N>6$, the simplified system is overdetermined. In this case, it needs to choose a suitable subsystem and compute its desired solution by the continuation method. For the cases $N=6$ and $N>6$, we develop two homotopy continuation methods, Algorithm 1 and Algorithm 2, to trace the moving object, respectively. Numerical results show that the new developed homotopy continuation method is robust, efficient and reliable for tracking the moving object. 


\section{Acknowledgement}

We would like to thank Professor Jubo Zhu from College of Science, National University of Defence Technology, China for providing the real data of a moving object and thank Professor Tien-Yien Li from MSU for valuable suggestions on homotopy continuation methods.

\section{Appendix}

Assume that $\mathbf{V}_{0}$ and $\mathbf{C V}_{0}$ are of full rank. We will show that, generally, $\widehat{\mathbf{A}}$ in (11) has rank $N-4$. From (11), we have

$$
\widehat{\mathbf{A}}=-\widehat{\mathbf{C}} \mathbf{V}_{0} \mathbf{A}+\widehat{\mathbf{C}}=\widehat{\mathbf{C}}\left(\mathbf{I}_{N}-\mathbf{V}_{0} \mathbf{A}\right) .
$$

Since $\mathbf{A} \mathbf{V}_{0}=\left(\mathbf{V}_{0}^{T} \mathbf{C}^{T} \mathbf{C} \mathbf{V}_{0}\right)^{-1}\left(\mathbf{V}_{0}^{T} \mathbf{C}^{T} \mathbf{C}\right) \mathbf{V}_{0}=\mathbf{I}_{3}$, it can be shown that the spectrum of $\mathbf{V}_{0} \mathbf{A}$, denoted by $\sigma\left(\mathbf{V}_{0} \mathbf{A}\right)$, is $\{1,1,1,0, \ldots, 0\}$. Therefore, $\sigma\left(\mathbf{I}_{N}-\mathbf{V}_{0} \mathbf{A}\right)=\{0,0,0,1, \ldots, 1\}$. Let $\widehat{\mathbf{V}}=\left[\mathbf{V}_{0}, \mathbf{e}\right] \in \mathbb{R}^{N \times 4}$. Since $\mathbf{C V}_{0}$ is of full rank, we have $\operatorname{rank}(\widehat{\mathbf{V}})=4$. It follows from the definitions of $\mathbf{A}$ and $\mathbf{e}$ that

$$
\widehat{\mathbf{A}} \widehat{\mathbf{V}}=\left[\widehat{\mathbf{C}}\left(\mathbf{I}_{N}-\mathbf{V}_{0} \mathbf{A}\right) \mathbf{V}_{0}, \widehat{\mathbf{C}}\left(\mathbf{I}_{N}-\mathbf{V}_{0} \mathbf{A}\right) \mathbf{e}\right]=\mathbf{0} .
$$

Hence, $\operatorname{rank}(\widehat{\mathbf{A}}) \leqslant N-4$.

Since $\mathbf{A} \in \mathbb{R}^{3 \times N}$ and $\operatorname{rank}(\mathbf{A})=3$, the dimension of $\mathcal{N}(\mathbf{A})$ is $N-$ 3 , where $\mathcal{N}(\mathbf{A})$ is the kernel of $\mathbf{A}$. It is easily seen that $\mathbf{e} \in \mathcal{N}(\mathbf{A})$. Let $\mathbf{U} \in \mathbb{R}^{N \times(N-4)}$ such that the set of column vectors of $\mathbf{U}$ form a basis of $\left\{\mathbf{x} \in \mathcal{N}(\mathbf{A}) \mid \mathbf{x}^{T} \mathbf{e}=0\right\}$. From (21), we have

$$
\widehat{\mathbf{A}} \mathbf{U}=\widehat{\mathbf{C}}\left(\mathbf{I}_{N}-\mathbf{V}_{0} \mathbf{A}\right) \mathbf{U}=\widehat{\mathbf{C}} \mathbf{U},
$$

where $\widehat{\mathbf{C}}=\left[\mathbf{0},-\mathbf{e}, \mathbf{I}_{N-2}\right]$. From (22) and $\mathbf{e}^{T} \mathbf{U}=\mathbf{0}$, we know that

there exists a vector $\mathbf{x}_{0}$ in the column space of $\mathbf{U}$ such that $\widehat{\mathbf{A}} \mathbf{x}_{0}=\mathbf{0}$ (it means $\operatorname{rank}(\widehat{\mathbf{A}})<N-4)$ if and only if $\hat{\mathbf{e}}=[-(N-1), 1, \ldots, 1]^{T}$ is in the column space of $\mathbf{U}$.

Since $\mathbf{A}=\left(\mathbf{V}_{0}^{T} \mathbf{C}^{T} \mathbf{C} \mathbf{V}_{0}\right)^{-1}\left(\mathbf{V}_{0}^{T} \mathbf{C}^{T} \mathbf{C}\right)$ and $\left(\mathbf{V}_{0}^{T} \mathbf{C}^{T} \mathbf{C} \mathbf{V}_{0}\right)$ is invertible, we obtain that $\mathbf{V}_{0}^{T} \mathbf{C}^{T} \mathbf{C U}=\mathbf{0}$. Then $\hat{\mathbf{e}}$ is in the column space of $\mathbf{U}$ if and only if $\mathbf{0}=\mathbf{V}_{0}^{T} \mathbf{C}^{T} \mathbf{C} \hat{\mathbf{e}}=\mathbf{V}_{0}^{T} \mathbf{C}^{T}[N, N, \ldots, N]^{T}=N \mathbf{V}_{0}^{T}[(N-1), 1 \ldots, 1]^{T}$, which is equivalent to $\mathbf{s}_{2}+\mathbf{s}_{3}+\cdots+\mathbf{s}_{N}=(N-1) \mathbf{s}_{1}$. Hence, we have the following results. 
Theorem 6.1. Assume that $\mathbf{V}_{0}$ and $\mathbf{C V}_{0}$ are of full rank. Then

(i). $\operatorname{rank}(\widehat{\mathbf{A}}) \leqslant N-4$, where $\widehat{\mathbf{A}}$ is defined in (11).

(ii). $\operatorname{rank}(\widehat{\mathbf{A}})<N-4$ if and only if $\mathbf{s}_{2}+\mathbf{s}_{3}+\cdots+\mathbf{s}_{N}=(N-1) \mathbf{s}_{1}$.

\section{References}

[1] E. L. Allgower and K. Georg, Continuation and path following. ACTA Numerica (1993), 1-64. MR1224680

[2] J. W. Armstrong, Low-frequency gravitational wave searches using spacecraft Doppler tracking. Living Rev. Relativity 9 (2006).

[3] B. Bertotti, G. Comoretto and I. Iess, Doppler tracking of spacecraft with multi-frequency links. Astron. Astrophys. 269 (1993), 608-611.

[4] M. E. Henderson and H. B. Keller, Complex bifurcation from real paths. SIAM J. Appl. Math. 50 (1990), 460-482. MR1043596

[5] H. B. Keller, Lectures on Numerical Methods in Bifurcation Problems. Springer-Verlag, Berlin, (1987). MR0910499

[6] T. Y. Li and X. Wang, Higher order turning points. Appl. Math. Comut. 64 (1994), 155-166. MR1298259

[7] T.-L. Lee, S.-S. Lin, W.-W. Lin, S.-T. Yau and J. Zhu, Polynomial calculations in doppler tracking. Commun. Inf. Syst. 12 (2012), 155182. MR3083690

[8] T. Y. Li, Solving polynomial systems by the homotopy continuation method, Handbook of numerical analysis, Vol. XI, 209-304, NorthHolland, Amsterdam (2003). MR2009773

[9] L. Nirenberg, Topics in Nonlinear Functional Analysis, Lecture notes, Courant Inst. Math. Sci. New-York University (2001). MR0488102

[10] S. N. Salinger and J. J. Brandstatter, Application of recursive estimation and Kalman filtering to Doppler tracking. IEEE Transactions on Aerospace and Electronic Systems 6 (1970), 585-592.

[11] J. Zhu, Private communication.

YUEH-CHENG KuO

Department of Applied Mathematics

NATiOnal University of KaOHSIUNG

KAOHSIUNG 811

TAIWAN

E-mail address: yckuo@nuk.edu.tw 
WEN-WEI LIN

Department of Applied Mathematics

National Chiao Tung University

HSINCHU 300

TAIWAN

E-mail address: wwlin@math.nctu.edu.tw

Shing-Tung Yau

DEPARTMENT OF MATHEMATICS

HARVARD UNIVERSITY

Cambridge, MA 02138

USA

E-mail address: yau@math.harvard.edu

Received March 17, 2014 